Abdullah Mohammed Kaleem* and Rajendra D. Kokate

\title{
An Efficient Adaptive Filter for Fetal ECG Extraction Using Neural Network
}

DOI 10.1515/jisys-2017-0031

Received February 3, 2017; previously published online September 5, 2017.

\begin{abstract}
Fetal electrocardiogram checking is a strategy for acquiring critical data about the state of the fetus during pregnancy and labor. This is done by measuring electrical signals created by the fetal heart as measured from multichannel potential recordings on the mother's body surface. In any case, extraction of fetal signal is difficult because the signal is marred by the mother's heartbeat signal. Subsequently, in this paper, a powerful versatile filtering strategy is utilized to eliminate the mother's heartbeat signal with the specific end goal of extricating the fetal signal. The proposed procedure was executed in the working stage of MATLAB and the execution results were investigated.
\end{abstract}

Keywords: Fetal electrocardiogram, heartbeat signal, neural network.

\section{Introduction}

Monitoring of fetal heart rate (FHR) patterns is a method developed for fetal reconnaissance and offers vital data about the soundness of the unborn [2]. Securing of fetal electrocardiogram (fECG) becomes a demanding task because immediate contact over the fetus can cause shock [8]. fECG waveform examination is performed with estimation of electrical movement from the fetal heart, and has been created in the course of the most recent three decades [19]. fECG can be measured by setting electrodes on the mother's midriff [1]. Nevertheless, it has low power and is mixed with a few sources of noise and interference [14].

The fECG flag stresses the fetal heart movement, which is the key to decide the FHR, fetal advancement, fetal development, presence of fetal pain or multiple pregnancies, and to prevent neonatal ailments and perform parametric examination of the fetal heart [8].

The fECG flag can be gained by using two strategies: the fetal scalp terminal (obtrusive) and maternal stomach area skin anode (non-invasive) [9]. The recorded stomach electrocardiogram flag contains three kinds of signs: maternal ECG (mECG) flag, fECG flag, and other obstruction signals (commotion) [9]. Notwithstanding the influence of maternal solid artifacts and electrical cable unsettling, the most grounded aggravation is $\mathrm{mECG}$, which is 5-10 times more grounded than fECG [19].

In any case, fECG signals are blacked out and become mixed with a few different sources of aggravation. The fetal scalp anode technique will yield a top-notch flag; however, it causes shock to the fetus. The scalp terminal must be set toward the head of the fetus while in the mother's womb, which is a painful practice that is risky for both the mother and the fetus [9].

A focal point is distinguishing diminished oxygen supply to the fetus, to allow early obstetric intervention to prevent fetal disorders or even mortality [3]. The fECG is utilized for detecting fetal cardiovascular recurrence and in predicting fetal acidosis [5]. However, fECG signals and different obstructions and artifacts are not generally stationarily blended and straightly divisible [14].

*Corresponding author: Abdullah Mohammed Kaleem, Department of Electronics and Telecommunication Engineering, MPGI School of Engineering, Nanded, Maharashtra, India, e-mail: abdullahmk6577@gmail.com

Rajendra D. Kokate: Department of Instrumentation Engineering, Government College of Engineering, Jalgaon, Maharashtra, India 
fECG basics were used to ascertain R-R beat intervals with a value of approximately $1 \mathrm{~ms}$ [17]. At present, observation of the fetus is totally focused on the heart rate. Currently, fECG examination is utilized as a part of the clinical work-up to investigate heart rate and associated factors [8]. Quick FHR estimation by phonocardiography guarantees a precise estimation of beats and pulsation interval [2].

One notable focus is the estimation of vacillations around the FHR gauge [3]. Versatile sifting is basic and quick, yet fetal ECG separated by this calculation still contains maternal ECG and other aggravations [19]. Versatile channels have the benefit that they can track the recurrence changes in the PLI, in this way lessening the commotion in biomedical applications [18].

Electrical clamors, which influence fECG signals, can be classified into the following types:

- mECG signal;

- Electrode contact noise;

- Motion artifact;

- Inherent noise in electronics equipment;

- Ambient noise [6].

\section{Related Work}

Immanuel et al. [7] isolated the ECG flag taken from skin terminals situated on a pregnant woman's body into the mECG flag and the fECG flag. Dazzle Source Separation was the system utilized for isolating these source signals. Independent component analysis (ICA) was used on the blended signs, and the isolated signs were reproduced using wavelet recreation.

Gustafson et al. [4] characterized whether maternal practice during pregnancy directs the impact of fetal breathing developments on the fetal cardiovascular autonomic control evaluated by measurements of heart rate (HR) and HR variability (HRV). Maternal practice altogether brings down the FHR, increases aggregate $\mathrm{HRV}$, and vagally intervenes with HRV with no distinction in recurrence band proportions. Critical communications between maternal practice and fetal breathing are found for measurements condensing all HRVs and a parasympathetic metric. Fetal breathing is connected with lost total HRV in the control group and no distinction in the exercise group. Both groups indicate improved vagal capacity during fetal breathing, more prominent in the exercise group. During in utero breathing developments, the fetus has improved cardiovascular autonomic capacity that may give the offspring a versatile preferred standpoint [12].

Chourasia et al. [2] depicted a non-intrusive, versatile, and reasonable antenatal care framework utilizing fetal phonocardiography (FPCG). The FPCG method can possibly allow minimal effort and long-term diagnostics to the underserved populace. The FPCG flag contains useful symptomatic data in regards to fetal well-being during the antenatal period. The FPCG signs are obtained from the maternal stomach surface by utilizing remote information procurement and a recording framework. The indicative parameters, e.g. pattern, inconsistency, and quickening and deceleration of the FHR, are obtained from the FPCG flag [16].

Morales et al. [13] represented the utilization of a reconfigurable framework for fECG estimation from the mother's gut ECG estimations. The framework depended on two diverse reconfigurable gadgets. At first, a field-programmable analog array gadget actualizes the simple reconfigurable preparation for ECG flag securing. The flag handling chain proceeds onto a field-programmable gate array (FPGA) gadget, which contains all the correspondence and interfacing conventions alongside particular advanced flag, preparing squares required for key period extraction from fECG waveforms. The cooperative energy between these gadgets provides the framework and the capacity to change any fundamental parameter during the procurement procedure for improving the outcome. The utilization of an FPGA permits to actualize the diverse calculations for fECG flag extraction, for example, versatile flag separation.

Karmakar et al. [10] explored the confirmation of FHR asymmetry and how the FHR asymmetry changes before and after 35 weeks of gestation. Non-intrusive fECG signals from 45 pregnant women at gestational age from 16 to 41 weeks with ordinary single pregnancies were broken down. A non-linear parameter called 
heart rate asymmetry (HRA) that measures the time asymmetry of the R-R interval time-arrangement flag was utilized to understand the progressions of HRA in early and late fetus groups.

Poian et al. [15] proposed a novel framework based on a compressive sensing theory, for the compression and joint detection/classification of mother and fetal heartbeats. Their scheme was based on the sparse representation of the components derived from ICA, which they proposed to apply directly in the compressed domain. Detection and classification were based on the activated atoms in a specifically designed reconstruction dictionary.

Lamesgin et al. [11] proposed a study that uses signals recorded from the mother's abdomen by a single ECG lead for efficient maternal QRS detection, fetal ECG extraction and enhancement, as well as FHR variability analysis by combining spectral analysis techniques with selected filters. The algorithm had been tested on 20 non-invasively recorded abdominal signals from the MIT/physioNet database, and the results found were very promising.

\section{Proposed Methodology}

The proposed strategy is aimed at successfully separating the fetal ECG motion from the corrupted environment. In this work, two signs were created by using two lead anodes: one to get the mid-region flag and the other to get the pulse flag. The mid-region lead catches the fetal ECG flag and the mid-section terminal catches the unadulterated pulse flag. The belly flag not only contains the fetal flag but it is also tainted by the heartbeat flag of the mother. Consequently, it is difficult to extract the fetal flag.

Subsequently, with a versatile commotion cancelation strategy, the ruined flag is sifted by using the method for keeping a referred clamor as reference information. A versatile channel is a channel that can selfalter the channel coefficients as indicated by an upgraded preparing calculation, which is driven by a backspread error flag. Here, as a preparation, a calculation double-stage neural system is utilized. The system has the capacity to manage complex flag handling circumstances more dependably than established versatile techniques. The proposed versatile clamor cancelation system is point by point in the accompanying area.

The proposed method was implemented on the working platform of MATLAB and the results were evaluated.

\subsection{Adaptive Filtering (Adaptive Noise Cancellation)}

A versatile channel is a channel that can self-alter the channel coefficients as per a streamlined preparation calculation that is driven by a back proliferated blunder flag. In a versatile clamor cancelation setup, the commotion adulterated unique flag will be contrasted as the reference flag. The contrast between the first flag degraded by clamor and the known reference flag (i.e. commotion flag) gives the desired flag. The distinction is named here as the mistake flag, which is to be minimized. The versatile channel is adjusted by the least mean square (LMS) mistake calculation, which is the most generally utilized versatile sifting calculation. In view of the LMS calculation, the versatile channel coefficients were adjusted until the mistake motion with minimum mean square error (MSE) is acquired.

The versatile commotion cancelation technique is shown in Figure 1.

In this proposed work, the versatile channel parameters are ideally chosen through the double-stage artificial neural network (ANN) method with less calculations, many-sided quality, and better productivity. In addition, the double-stage ANN upgrades the weight in view of the learning rate and force (weight of past cycle). The versatile channel separates the fetal flag with the assistance of double-stage ANN via consequently getting the suitable parameters. The proposed versatile commotion cancelation method utilizing double-stage ANN is shown in Figure 2.

In Figure 2, $M(S)=$ abdominal signal, $R(S)=$ reference signal (chest signal), $d(s)=$ chest signal with delay, $\hat{d}(s)=$ trained chest signal with delay, and $E(S)=$ extracted fetal signal (error signal). 


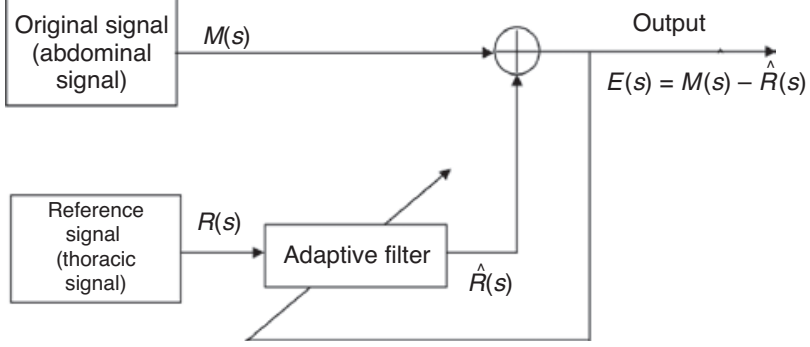

Figure 1: Adaptive Noise Cancellation Method.

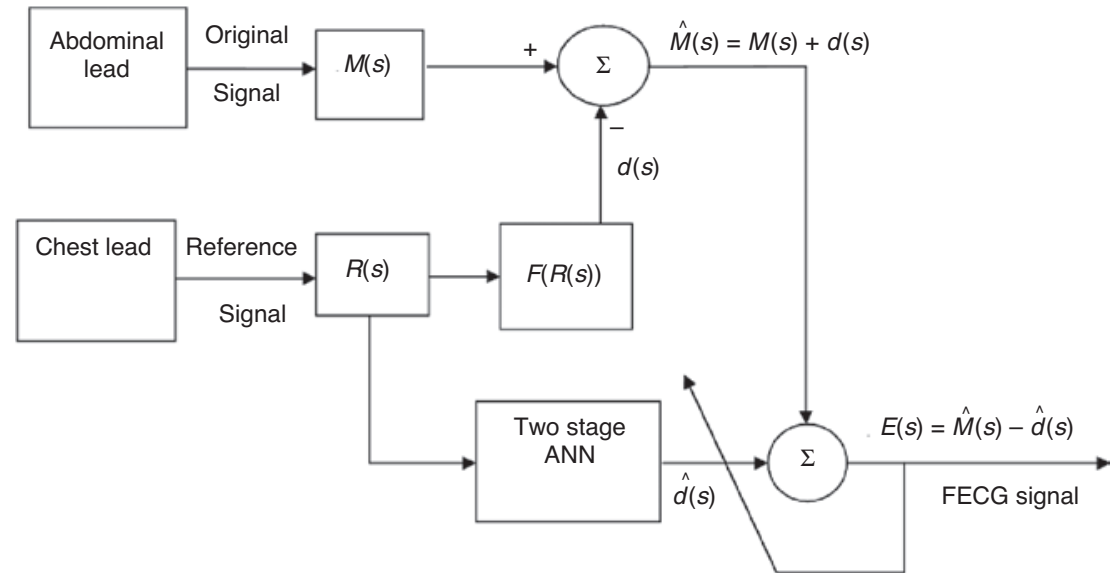

Figure 2: Proposed Adaptive Noise Cancellation Method.

\subsubsection{Input Components for Adaptive Noise Cancellation}

Two inputs, such as the abdominal signal as well as the reference thoracic signals, were taken. Let us consider if the reference thoracic signal and the abdominal signal are $r(j)$ and $f(j)$, respectively. The input signal $f(j)$ contains a noise source, $n_{0}(j)$.

Hence, the original input signal (abdomen signal) can be given as

$$
M(S)=f(j)+n_{0}(j) .
$$

Moreover, the reference input signal (maternal heartbeat signal) can be given as

$$
R(S)=n_{1}(j),
$$

where $n_{0}(j)$ and $n_{1}(j)$ are the noise sources. The noise source $n_{0}(j)$ is an unknown component. Removal of the noise component in order to yield the fetal ECG signal is a complex procedure. By means of the adaptive noise cancellation procedure, input signal $M(S)$ is compared with the known noise source, $n_{1}(j)$, and the difference output is attained.

The noise sources $n_{0}(j)$ and $n_{1}(j)$ were associated with each other in some way that they should be equal. The difference output (error signal) is the fetal ECG signal $(f(j))$.

The error signal should converge to the signal $f(j)$ (i.e. fetal signal), but not converge to the exact signal (i.e. abdomen signal). The extracted signal is examined by using the least MSE value. The adaptive filter coefficients adapt to cause the error signal to be a noiseless version of the signal, $f(j)$. Here, the adaptive filter coefficients were adjusted automatically until the clear fetal signal is extracted.

In this proposed work, the adaptive filter parameters are optimally carefully chosen through the dualstage ANN technique with less computation complexity and better efficiency. 


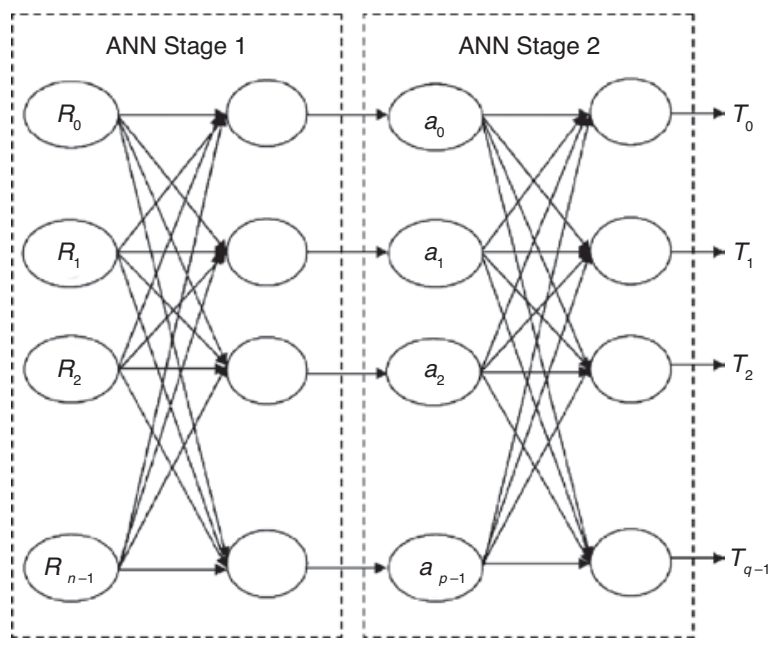

Figure 3: Dual-Stage ANN.

\subsection{Two-Stage ANN}

The versatile channel coefficients are chosen by using the method for double-stage simulated neural system strategy. In the two-phase ANN method, the preparation is done in two phases. The ANN comprises three layers: input layer, concealed layer, and yield layer.

In this mode, the input factor to stage I is the reference maternal flag that will be prepared at first with a deferral. Then, keeping in mind the end goal of making the obscure commotion source like the known clamor source, a deferral is added to the maternal flag. As the pulse flag is not added specifically to the mid-region flag, it is included with a postponement. The execution of preparation is assessed by the method for the MSE. At that point, the prepared yield from the stage I neural system is used to prepare the stage II neural system.

The design of double-stage ANN is shown in Figure 3.

For preparing, the yield of stage I is provided at the contribution of stage II, and the contribution of stage I is dealt with as the yield for stage II. At that point, the BPN calculation is executed utilizing this given information yield connection. Finally, the stage II neural system is prepared until the yield is accomplished without mistake (implying that the yield of stage II must be equivalent to the contribution of stage I). In the two-stage ANN, no changes have been made for cost functions, the input factor to stage I is the reference maternal flag prepared at first stage of ANN, and the pulse flag is included with a postponement of ANN.

The process taking place within each layer of the ANN technique is given in the following.

Input unit: The input $R$ [i.e. known noise, $n_{1}(j)$ ] is the reference maternal heartbeat signal. Here, the input signal $R$ with a delay $n$ is well thought out.

Hidden unit: The process carried out in the hidden unit is presented as follows:

$$
R_{j}^{m+1}(l)=f\left\{b_{j}+\sum_{k=0}^{M} w_{k j}^{m} R_{k}^{m}(l-n)\right\}
$$

where $R_{j}^{m+1}(l)$ is the output in layer $m$ at time $l$ and $R_{k}^{m}(l-n)$ is the delayed input layer $m$ at time $l$.

Furthermore, $f(x)$ is the activation function of the hidden layer. The activation function is the tan-sigmoid function as provided below:

$$
f(x)=\frac{2}{1+e^{-2 x}}-1
$$


Output unit: The computations accomplished in the output unit can be provided as follows:

$$
T=\sum_{k=1}^{M} W_{k} R_{\text {hidden }} \text {. }
$$

At each emphasis, the heaviness of the system will be upgraded. Once the preparation procedure is finished, the last weight is taken and given to the versatile channel. The channel parameters are then balanced as per the weights obtained.

Finally, the distinction between the reference flag and the stomach flag is taken to extricate the fetal flag. The separated fetal flag is investigated by using the method for registering the MSE estimation of the fetal flag.

\section{Results and Discussions}

The proposed system was actualized in the working stage of MATLAB and the execution results were dissected. The achieved results and the following discussion on their premise are presented in this section. The routine strategy throughputs are contrasted and the untried proposed strategy is a versatile clamor cancelation method for development reason.

Biomedical signal means a collective electrical signal acquired from any organ that represents a physical variable of interest. Tapped delay line is a function of time and is describable in terms of its amplitude, frequency, and phase. fECG is a biomedical signal that gives electrical representation of FHR to obtain the vital information about the condition of the fetus during pregnancy and labor from the recordings on the mother's body surface. The fECG signal is a comparatively weak signal $(<20 \%$ of the mECG) and often embedded in noise. The FHR lies in the range from 1.3 to $3.5 \mathrm{~Hz}$, and sometimes it is possible for the $\mathrm{mECG}$ and some of the fECG signals to be closely overlapping. To verify the feasibility of the proposed method, experiments were conducted using 10 cases of clinical data from physioNet database. All the cases are the combinations of abdominal signals and thoracic signals.

\subsection{Signal-to-Noise Ratio}

Signal-to-noise ratio (SNR) is utilized to gauge the level of a coveted flag to the level of commotion emerging in the circuits. It is characterized as the proportion of flag energy to the clamor control, frequently communicated in decibels. The higher the estimation of SNR, the more prominent is the flag than the clamor. SNR is obtained by using the following formula:

$$
\mathrm{SNR}=\frac{P_{\text {signal }}}{P_{\text {noise }}} .
$$

The graph of SNR for both the existing and the proposed techniques for a series of input samples are shown in Figure 4, and the tabulation for the same is shown in Table 1.

As conferred, the greater the SNR value, the better is the signal strength. The proposed method is an adaptive noise cancellation technique that has considerably better performance than the existing technique (neural network) to filter out the noise while extracting fECG. From the tabulation, it can be noted that for every sample, the SNR in the proposed technique is consistently higher than the existing one.

\subsection{MSE}

The root MSE is also named as root mean square deviation. It is used to measure the differences between values foretold by an estimator and the values actually observed. The MSE embodies the sample standard 


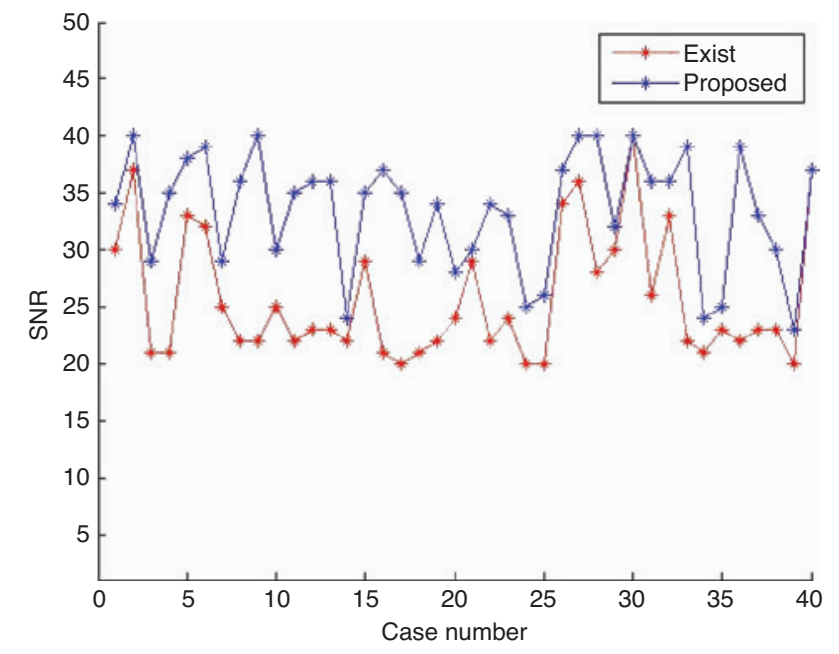

Figure 4: SNR Graph.

Table 1: Tabulation for SNR.

\begin{tabular}{lrr}
\hline SNR & & \\
\hline Case no. & $\begin{array}{r}\text { Existing technique } \\
\text { (neural network) }\end{array}$ & $\begin{array}{r}\text { Proposed } \\
\text { technique }\end{array}$ \\
\hline 1 & 30 & 34 \\
2 & 37 & 40 \\
3 & 21 & 29 \\
4 & 21 & 35 \\
5 & 33 & 38 \\
6 & 32 & 39 \\
7 & 25 & 29 \\
8 & 22 & 36 \\
9 & 22 & 40 \\
10 & 25 & 30 \\
\hline
\end{tabular}

deviation of the differences between predicted values and observed values. The MSE is used to average the magnitudes of errors in predictions. The MSE should decrease to some a small, stable value:

$$
\mathrm{MSE}=\frac{1}{m} \sum_{i=1}^{m}\left(\hat{X}_{i}-X_{i}\right)^{2},
$$

where $\hat{X}$ is the vector of $m$ prediction and $X$ is the vector of perceived value corresponding to inputs.

Figure 5 and Table 2 provide the MSE data, with notable comparisons between the existing technique and the proposed technique.

The MSE graph for the existing technique and the proposed method for an adaptive noise cancellation technique is depicted in Figure 5. The existing technique shows large MSEs at the end, which indicates inconsistencies between training samples, whereas the proposed method shows consistency at the end between the training samples, which indicates the reasonability of the implemented technique.

The deviation readings for common input samples are depicted in Table 2 for both the existing and proposed techniques. From the tabulation for the proposed technique, with every iteration, the MSE deviation decreases from the previous value. 


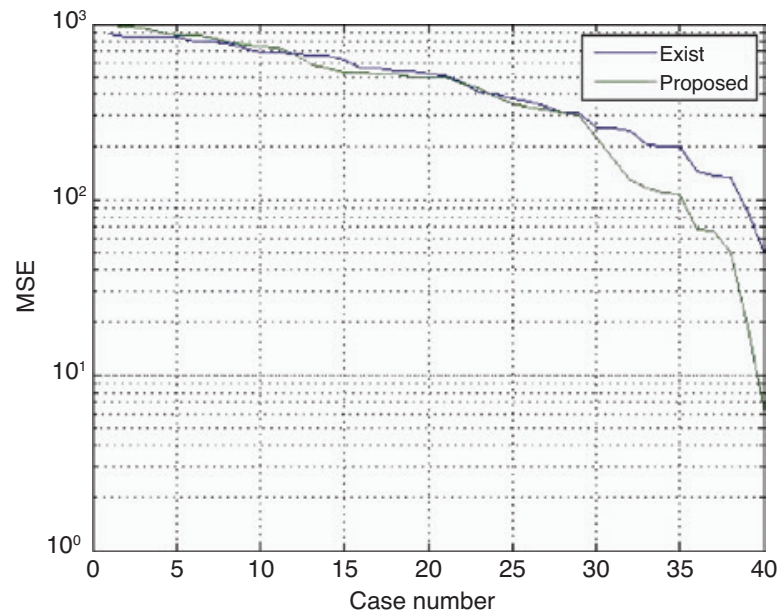

Figure 5: MSE Graph.

Table 2: Tabulation for MSE.

\begin{tabular}{lrr}
\hline MSE & \\
\hline Case no. & $\begin{array}{r}\text { Proposed } \\
\text { technique }\end{array}$ & $\begin{array}{r}\text { Existing } \\
\text { technique }\end{array}$ \\
\hline 1 & 0.885 & 0.981 \\
2 & 0.841 & 0.975 \\
3 & 0.839 & 0.959 \\
4 & 0.839 & 0.887 \\
5 & 0.834 & 0.857 \\
6 & 0.797 & 0.853 \\
7 & 0.786 & 0.84 \\
8 & 0.778 & 0.791 \\
9 & 0.729 & 0.766 \\
10 & 0.689 & 0.74 \\
\hline
\end{tabular}

\subsection{Extraction of fECG Signal from the Abdominal Signal}

To extract the fECG signal from our proposed algorithm, the experimental result of the proposed algorithm is tested on input sample data. For constructing the training data, each of the thoracic and abdomen signals can be framed with overlapping signals. We can synthesize the thoracic and abdominal signals for comparing the performance of the proposed algorithm. The three waveforms from top to bottom are the simulated abdominal signal, the simulated mECG, and the extracted fECG. It can be observed that the proposed method could remove mECG and extract fECG efficiently. Naturally, the fECG amplitude is less than that of the mECG; however, the fetal heart beats faster than the maternal heart. The maternal signal is aligned with the maternal component of the abdominal signal. fECG is extracted by subtracting the aligned maternal signal with the abdominal signal. The extracted fECG in all cases may be given a better visual judgment. This implies that the proposed system outperforms the existing one. The representative results for different cases are shown in Figures 6-11.

The abdominal ECG signal and fECG signal obtained for input samples 1 and 2 are shown in Figures 6 and 7, respectively. It can be observed that the result of fECG extraction is markedly improved, and both mECG and other disturbances are eliminated.

For constructing the training data, each of the thoracic and abdominal signal can be framed with overlapping signals. We can synthesize the thoracic and abdominal signals for comparing the performance of the 

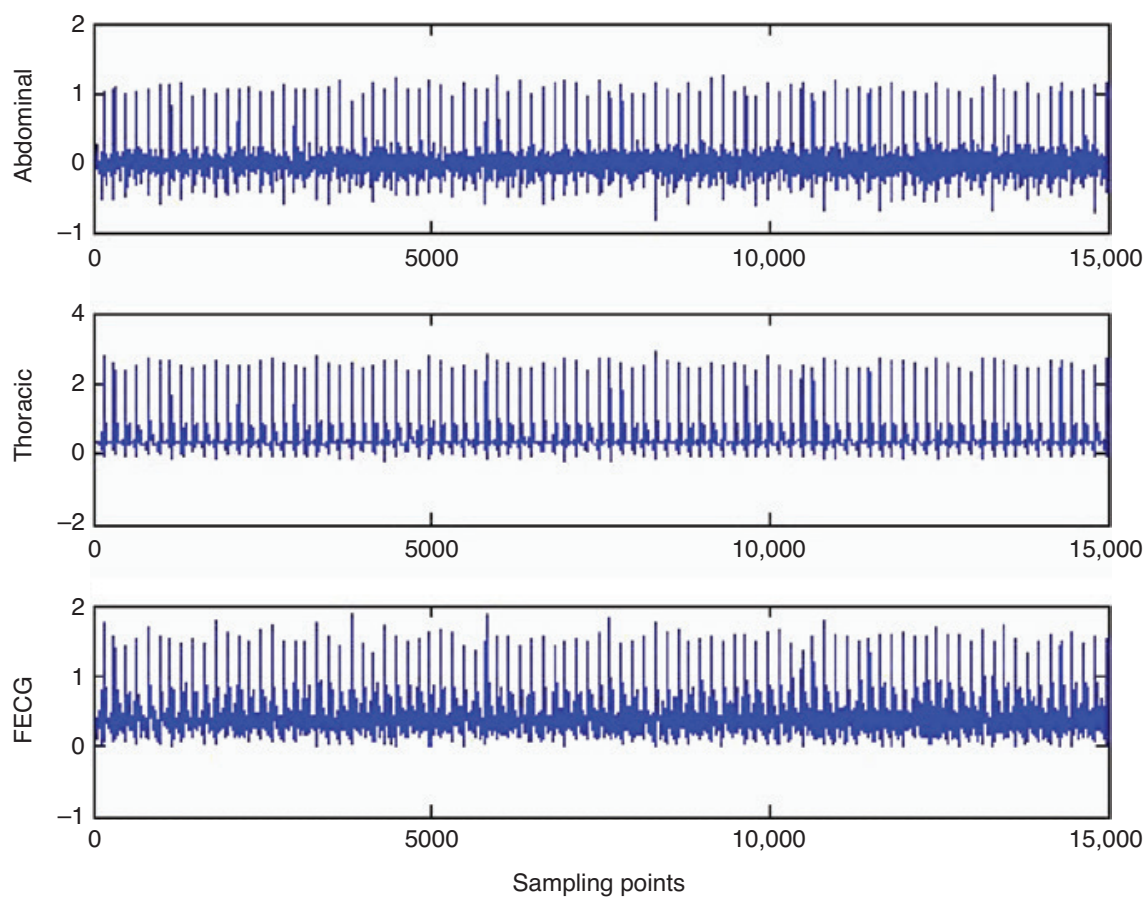

Figure 6: fECG Extraction for Sample Input 1.
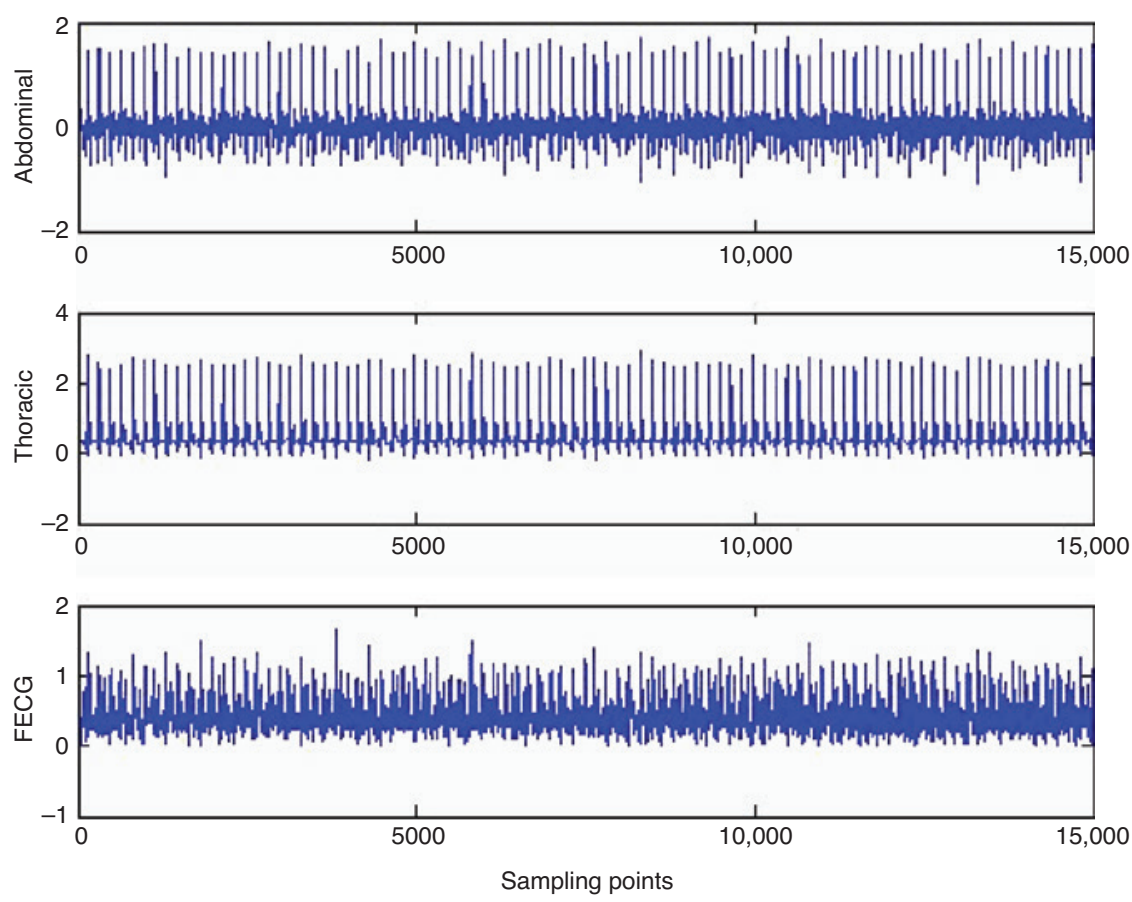

Figure 7: fECG Extraction for Sample Input 2.

proposed algorithm. Figures 8 and 9 show the results of the proposed algorithm for sample inputs 3 and 4, respectively. It can be observed that the proposed method could remove $\mathrm{MECG}$ and extract fECG efficiently.

Figures 10 and 11 show the results of the proposed algorithm for sample inputs 5 and 6, respectively. In Figures 10 and 11, multiple reference channels are designated to increase the extraction accuracy. It can be observed that the estimated fECG signal is much cleaner for an iteration range. 

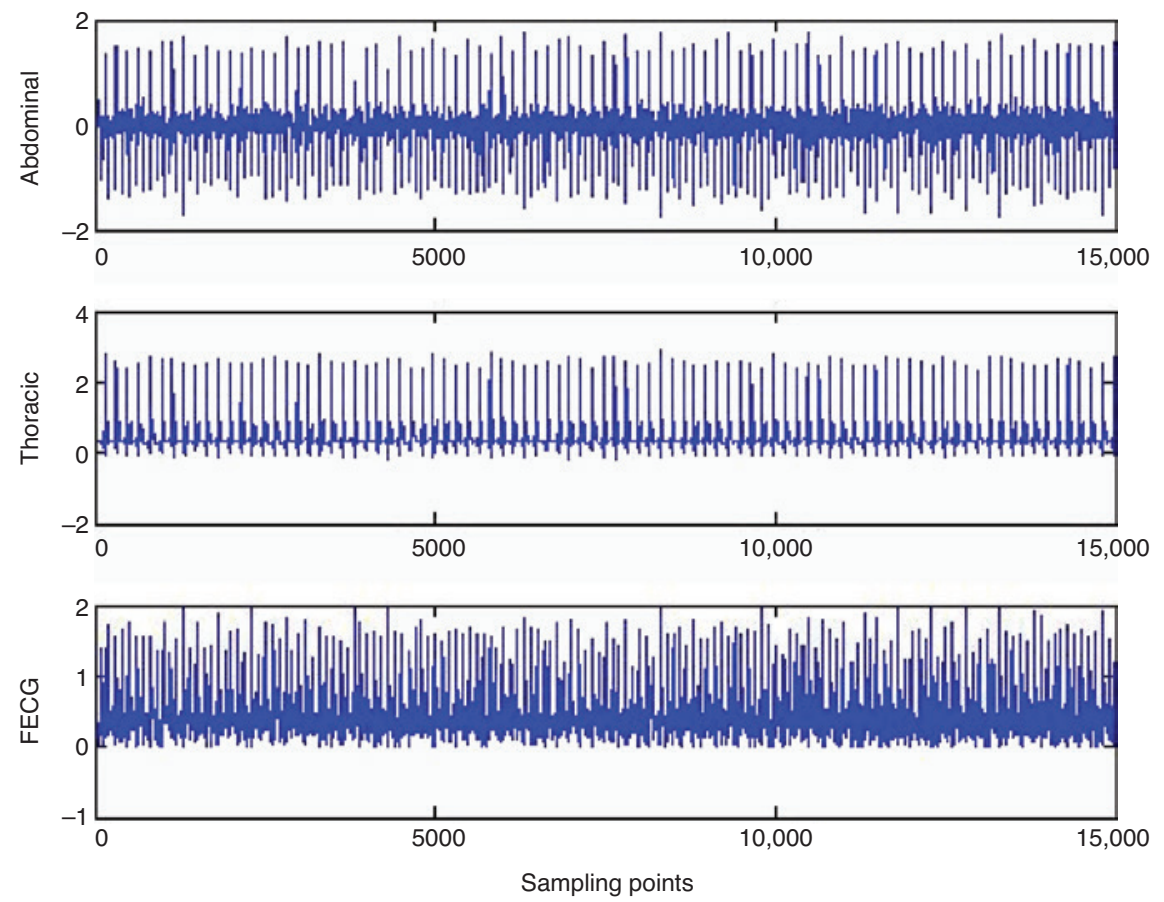

Figure 8: fECG Extraction for Sample Input 3.
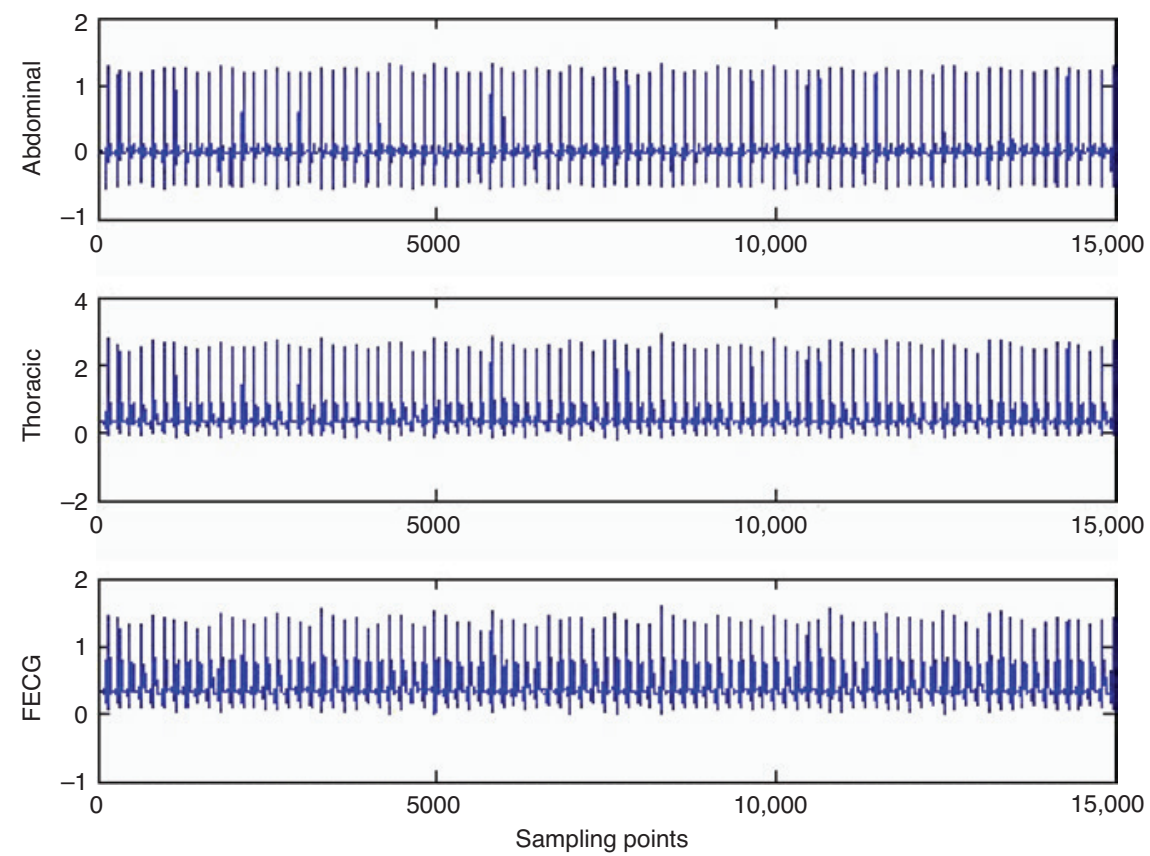

Figure 9: fECG Extraction for Sample Input 4.

\section{Conclusion}

In this paper, we have used a new technique for extraction of fECG with better efficiency over the existing technique. The extraction of $\mathrm{EECG}$ is critical from a clinical perspective to obtain tried and true information on fetal status, to recognize irregularities, to monitor the fetus during pregnancy, to enable measurements 

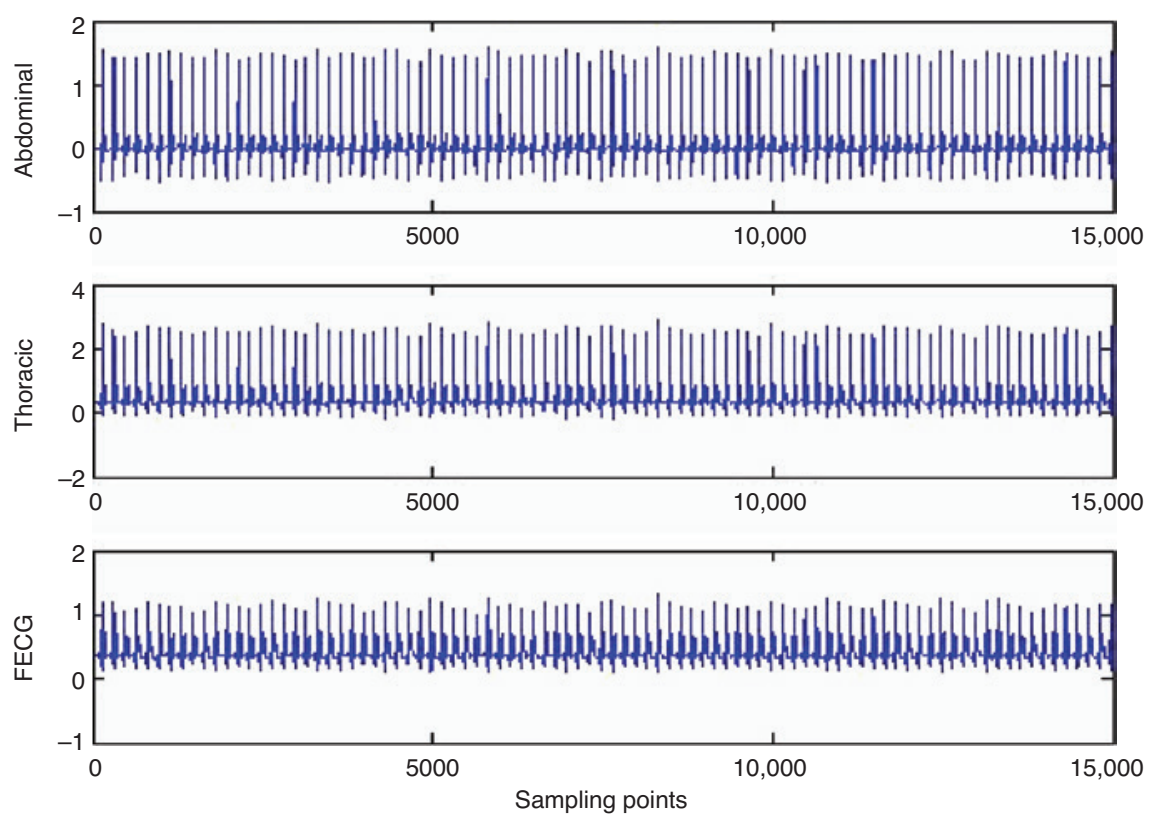

Figure 10: fECG Extraction for Sample Input 5.
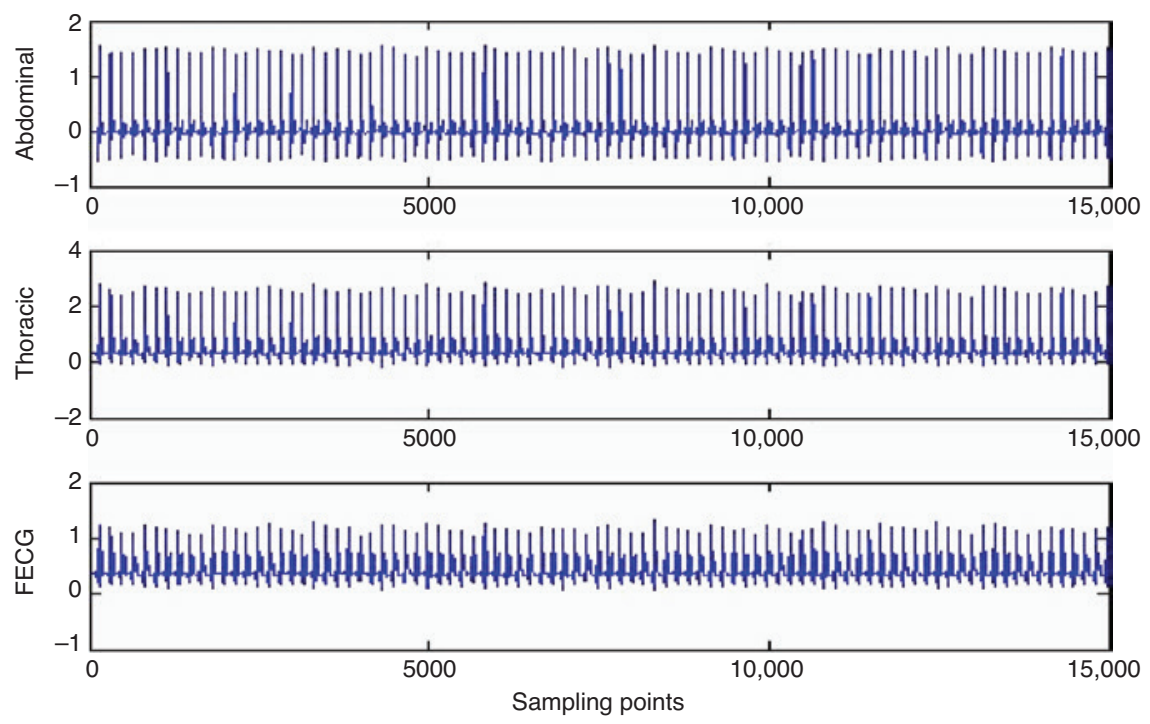

Figure 11: fECG Extraction for Sample Input 6.

for ensuring fetal success, to check whether the fetus is alive or dead, and to detect twin pregnancies. In this proposed strategy, a productive versatile clamor cancelation system utilizing a double-stage neural system is used. The quality of the extricated fetal flag is likewise examined by using the method for assessing MSE and SNR. The execution results are in contrast to the current procedures, which demonstrates the proficiency of the proposed strategy.

\section{Bibliography}

[1] J. L. Camargo-Olivares, R. Martín-Clemente, S. Hornillo-Mellado, M. M. Elena and I. Román, The maternal abdominal ECG as input to MICA in the fetal ECG extraction problem, IEEE Signal Process. Lett. 18 (2011), 161-164. 
[2] V. S. Chourasia, A. K. Tiwari and R. Gangopadhyay, A novel approach for phonocardiographic signals processing to make possible fetal heart rate evaluations, Digital Signal Process. 30 (2014), 165-183.

[3] M. D. Costaa, W. T. Schnettler, C. Amorim-Costa, J. Bernardes, A. Costa, A. L. Goldberger and D. Ayres-de-Campos, Complexity-loss in fetal heart rate dynamics during labor as a potential biomarker of acidemia, Early Hum. Dev. 90 (2014), 67-71.

[4] K. M. Gustafson, L. E. May, H.-W. Yeh, S. K. Million and J. J. B. Allen, Fetal cardiac autonomic control during breathing and non-breathing epochs: the effect of maternal exercise, Early Hum. Dev. 88 (2012), 539-546.

[5] L. Han, X.-J. Pu and X.-J. Chen, Method of fetal electrocardiogram extraction based on n-support vector regression, Proc. Res. Art. IET Signal Process. 9 (2015), 430-439.

[6] M. A. Hasan, M. I. Ibrahim and M. B. I. Reaz, Fetal ECG extraction from maternal abdominal ECG using neural network, J. Softw. Eng. Appl. 2 (2009), 330-334.

[7] J. J. R. Immanuel, V. Prabhu, V. J. Christopheraj, D. Sugumar and P. T. Vanathi, Separation of maternal and fetal ECG signals from the mixed source signal using FASTICA, in: Proceedings of International Conference on Communication Technology and System Design, 2011.

[8] D. J. Jagannath and A. I. Selvakumar, Issues and research on foetal electrocardiogram signal elicitation, Biomed. Signal Process. Control 10 (2014), 224-244.

[9] D. J. Jagannath and A. I. Selvakumar, Superior foetal electrocardiogram signal elicitation using a novel artificial intelligent Bayesian methodology, Appl. Soft Comput. 37 (2015), 1002-1017.

[10] C. Karmakar, Y. Kimura, M. Palaniswami and A. Khandoker, Analysis of fetal heart rate asymmetry before and after 35 weeks of gestation, Biomed. Signal Process. Control 21 (2015), 43-48.

[11] G. Lamesgin, Y. Kassaw and D. Assefa, Extraction of fetal ECG from abdominal ECG and heart rate variability analysis, Adv. Intell. Syst. Comput. 334 (2015), 65-76.

[12] C. Maier and H. Dickhaus, Extraction of respiratory myogram interference from the ECG and its application to characterize sleep-related breathing disorders in atrial fibrillation, J. Electrocardiol. 47 (2014), 826-830.

[13] D. P. Morales, A. García, E. Castillo, M. A. Carvajal, L. Parrilla and A. J. Palma, An application of reconfigurable technologies for non-invasive fetal heart rate extraction, Med. Eng. Phys. 35 (2013), 1005-1014.

[14] M. Niknazar, B. Rivet and C. Jutten, Fetal ECG extraction by extended state Kalman filtering based on single-channel recordings, IEEE Trans. Biomed. Eng. 60 (2013), 1345-1352.

[15] G. D. Poian, R. Bernardini and R. Rinaldo, Separation and analysis of fetal-ECG signals from compressed sensed abdominal ECG recordings, IEEE Trans. Biomed. Eng. 63 (2016), 1269-1279.

[16] K. Prasanth, B. Paul and A. A. Balakrishnan, Fetal ECG extraction using adaptive filters, Int. J. Adv. Res. Elect. Electron. Instrum. Eng. 2 (2013), 1483-1487.

[17] T. Stampalija, D. Casati, M. Monticoc, R. Sassi, M. W. Rivolta, V. Maggi, A. Bauer and E. Ferrazzi, Parameters influence on acceleration and deceleration capacity based on trans-abdominal ECG in early fetal growth restriction at different gestational age epochs, Eur. J. Obstet. Gynecol. Reprod. Biol. 188 (2015), 104-112.

[18] D. D. Taralunga, I. Gussi and R. Strungaru, Fetal ECG enhancement: adaptive power line interference cancellation based on Hilbert Huang Transform, Biomed. Signal Process. Control 19 (2015), 77-84.

[19] S. Wu, Y. Shen, Z. Zhou, L. Lin, Y. Zeng and X. Gao, Research of fetal ECG extraction using wavelet analysis and adaptive filtering, Comput. Biol. Med. 43 (2013), 1622-1627. 